\title{
Inhalt
}

Seite

Vorwort

$\mathrm{V}$

\section{In memoriam Christoph Trzaskalik}

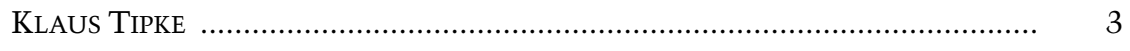

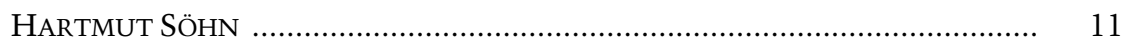

\section{Allgemeines Steuerrecht, Finanzverfassung}

\section{RÜDIGER VON GROLL}

Die Mißachtung der Abgabenordnung, ein Beitrag zur

Verunsicherung im Steuerrecht - dargestellt am Beispiel der

Tatbestandsverwirklichung

MONIKA JACHMANN

Gemeinnütziger Sport versus subventionierte Freizeitbetätigung ......

PETER FISCHER

Gemeinwohl, Daseinsvorsorge und bürgerschaftliches Engagement eine Gedankenskizze zum Zweckbetrieb (§ $65 \mathrm{AO}$ )

JOACHIM N. STOLTERFOHT

Bedarf ein feststellender Verwaltungsakt einer speziellen

Ermächtigungsgrundlage?

KLAUS-Dieter DRÜEN

Bestimmtheitserfordernis und Nichtigkeitsfolge bei Steuer-

bescheiden

KLAUS TIPKE

$\S 160 \mathrm{AO}$ - nochmals systematisch überdacht

\section{KLAUS SIEKER}

Im Wesentlichen unverwertbare Aufzeichnungen zu internationalen Verrechnungspreisen im Sinne von $\S 162$ Abs. 3 und 4 AO

JOACHIM SCHULZE-OSTERLOH

Der Steuerbescheid nach $\S 167$ Abs. 1 Satz 1 AO gegen den

Haftungsschuldner 
Inhalt

HANS GEORG RUPPE

Vertrauensschutz und Verwaltungserlässe

HEINRICH WILHELM KRUSE

Über Dienstleistungspflichten und Haftung

LERKE OSTERLOH

Die Einheitlichkeit der Lebensverhältnisse als offene Frage der

Finanzverfassung

III. Einkommensteuer, Ertragsteuern

Sebastian MÜLler-Franken

Verfassungsrecht und Einkommensteuerrecht

JOHANNA HEY

Das Individualsteuerprinzip in Einkommen-, Körperschaft- und

Gewerbesteuer

HeINZ-JÜRGEN PEZZER

Vermietung und Verpachtung - eine strukturell defizitäre

Einkunftsart

Rolf PefFekoven

Zur Besteuerung des selbstgenutzten Wohneigentums - Plädoyer

für die Investitionsgutlösung -

WERNER DORALT

Betriebsaufgabe durch Verpachtung?

WALTER DRENSECK

Einkommensteuerreform und objektives Nettoprinzip

KURT JOACHIM VON BORNHAUPT

Abstands-, Räumungs- und Erhaltungsaufwendungen des

Eigentümers bei Beendigung der Vermietungsphase

JÖRG GILOY

Zum Zeitpunkt der steuerlichen Erfassung von Einnahmen und

Ausgaben als technisches und wertendes Prinzip

FRANZ WASSERMEYER

Über den Zufluss fiktiver Beteiligungserträge vor dem Hintergrund des BMF-Schreibens vom 15. Juli $2004 \mathrm{zu} \S 8 \mathrm{a} \mathrm{KStG}$ 


\section{DIETER BIRK}

Rückzahlbare Steuern und hinausgeschobene Steuererstattungen Zur kompetenzrechtlichen Zulässigkeit des sog. Körperschaftsteuermoratoriums

IV. Andere Steuern, Lenkungsabgaben

WERNER WIDMANN

Die Verlagerung der Umsatzsteuerschuld auf den Leistungsempfänger als Beispiel der Steuererhebungspflichten Privater

RAINER HÜTTEMANN

Die Neuregelung der Rundfunkbesteuerung

PAUL KIRCHHOF

Lenkungsteuern

Peter Selmer

Ökologische Steuerreform, Verfassungsrecht und Bundesverfassungsgericht

ANNE WoHLFeIL/CHRISTIAN KAESER

Lenkung durch Gebühren

\section{Steuerstrafrecht}

\section{ROMAN SEER}

Steueramnestie und Idee einer Entpönalisierung des Steuerrechts

\section{WOLFRAM REIß}

Auswirkungen der Unschuldsvermutung aus Art. 6 Abs. 2 der EMRK im Steuerrecht

Liste der Veröffentlichungen von Christoph Trzaskalik 507

Autorenverzeichnis

Stichwortverzeichnis 
